\title{
ESTUDO ANATÔMICO DO TRAJETO DO NERVO MUSCULOCUTÂNEO EM RELAÇÃO AO PROCESSO CORACOIDE
}

\author{
ANATOMICAL STUDY OF THE MUSCULOCUTANEOUS NERVE \\ IN RELATION TO THE CORACOID PROCESS
}

Fabiano Rebouças ${ }^{1}$, Romulo Brasil Filho², Cantidio Filardis ${ }^{1}$, Renato Rodrigues Pereira ${ }^{3}$, Alessandro Alvarenga Cardoso ${ }^{4}$

\section{RESUMO}

Objetivo: Os autores realizaram o estudo anatômico do trajeto do nervo musculocutâneo pela dissecção de 20 ombros em 10 cadáveres adultos frescos. Método: Mediu-se a distância da borda inferior do processo coracoide, ao ponto de penetração do ramo mais proximal do nervo musculocutâneo no músculo coracobraquial, denominada base. Partindo da borda inferomedial do processo coracoide, foi medida uma segunda distância até o ponto em que o fascículo lateral do plexo braquial cruza o músculo subclávio, sendo identificada como altura. A terceira mensuração foi da área triangular formada pelas duas primeiras medidas, denominada área. Resultados: Observou-se que a média da base foi de 3,42cm, com variações de 2,38 a 4,30cm. A medida da altura foi em média $2,75 \mathrm{~cm}$, variando entre 1,03 a $3,80 \mathrm{~cm}$, e a média da área foi de 4,92 $\mathrm{cm}^{2}$, variando entre 1,22 a 7,99 $\mathrm{cm}^{2}$. Conclusão: Estas medidas são de grande importância, devido ao risco de lesão do nervo musculocutâneo nas abordagens cirúrgicas do ombro.

Descritores - Nervo musculocutâneo/anatomia \& histologia; Nervo musculocutâneo/lesões; Ombro/cirurgia

\section{ABSTRACT}

Objective: The authors performed an anatomic study of the trajectory of the muscle cutaneous nerve, dissecting 20 shoulders in 10 fresh adult corpses. Method: The distance was measured from the inferior edge of the coracoid process to the point of penetration of the nearest branch of the cutaneous nerve muscle of the coracobrachialis muscle, called base. Starting at the inferior-medial edge of the coracoid process, a second measurement was made to the point at which the lateral fascicle of the brachial plexus crosses the subclavius muscle, denominated height. The third measurement was of the triangular area formed by the two first measurements, denominated area. Results: The average base length was $3.42 \mathrm{~cm}$, varying from $2.38 \mathrm{~cm}$ to $4.40 \mathrm{~cm}$. The height measurement was $2.74 \mathrm{~cm}$, on average, varying between $1.03 \mathrm{~cm}$ and $3.80 \mathrm{~cm}$. And the average area was $4.92 \mathrm{~cm}^{2}$, varying between $1.22 \mathrm{~cm}^{2}$ and 7.99 $\mathrm{cm}^{2}$. Conclusion: These measurements are very important due to the risk of injury in the cutaneous nerve muscle in surgeries performed on the shoulder.

Keywords - Musculocutaneous nerve/anatomy \& histology; Musculocutaneous nerve/Injuries; Shoulder/surgery

\section{INTRODUÇÃO}

O nervo musculocutâneo é um dos ramos do fascículo lateral do plexo braquial. As fibras que o constituem procedem do quinto e sexto pares cervicais, e algumas do sétimo. Inicia seu trajeto atrás do músculo peitoral menor, acima e lateralmente ao nervo mediano e à ar- téria axilar. Dirige-se obliquamente para baixo e lateral, cruzando acima do tendão do músculo subescapular e em seguida penetrando o músculo coracobraquial. Ao sair deste músculo, percorre entre o músculo bíceps, à frente, e o músculo braquial, por detrás, atravessando diagonalmente a face anterior do braço, chegando à sua

\footnotetext{
1 - Médico Assistente do Grupo de Ombro e Cotovelo do COT do Hospital do Servidor Público Estadual - São Paulo, SP.

2 - Chefe do Grupo de Ombro e Cotovelo do COT do Hospital do Servidor Público Estadual - São Paulo, SP.

3 - Ex-Residente do COT do Hospital do Servidor Público Estadual - São Paulo, SP.

4 - Estagiário do Grupo de Ombro e Cotovelo do COT do Hospital do Servidor Público Estadual - São Paulo, SP.
}

Trabalho realizado pelo Grupo de Ombro e Cotovelo do Serviço de Ortopedia e Traumatologia do Hospital do Servidor Público Estadual - COT/HSPE. Correspondência: Centro de Estudos Ortopédicos - HSPE/SP - Rua Borges Lagoa, 1.755, 1ª andar, sala 180 - Vila Clementino -04038-034 - São Paulo, SP. E-mail: aac.jf@hotmail.com 
face lateral, entre o músculo braquiorradial e o extremo inferior do músculo bíceps. No cotovelo, perfura a aponeurose superficial, medialmente a veia cefálica, na altura da interlinha articular, onde torna-se subcutâneo, e se ramifica pela face lateral do antebraço $^{(1)}$.

Em seu curso, o nervo musculocutâneo origina duas classes de ramos: colaterais e terminais. No braço originam-se ramos colaterais para os músculos coracobraquial, bíceps e braquial, ramo para a diáfise do úmero, ramos vasculares e um ramo articular para face anterior do cotovelo. Os ramos terminais surgem quando o nervo musculocutâneo se torna subcutâneo, sendo um anterior e outro posterior. O ramo posterior, mais lateral, passa atrás da veia cefálica, alcança sucessivamente as camadas externas e posteriores do antebraço e descem até o carpo, emitindo ramos para pele. $\mathrm{O}$ ramo anterior continua na direção descendente, à frente da veia cefálica, avançando para a face anterior do antebraço e terminando como pequenos ramos destinados à pele da região anterolateral do antebraço ${ }^{(1)}$.

Diversos procedimentos cirúrgicos do ombro envolvem mobilizações ou afastamentos dos músculos que se inserem no processo coracoide. Estas cirurgias incluem osteotomias e transferências do processo coracoide, laçadas subcoracoides e transferências musculares. A lesão do nervo musculocutâneo, como complicação das cirurgias do ombro, foi o que nos motivou a realizar o seu estudo anatômico ${ }^{(2-9)}$.

\section{MÉTODO}

Vinte ombros de 10 cadáveres frescos foram dissecados utilizando-se a via deltopeitoral de $15 \mathrm{~cm}$. Após dissecção da pele e do tecido celular subcutâneo, realizou-se desinserção do músculo peitoral maior, para melhor exposição dos músculos coracobraquial e peitoral menor (Figura 1); o músculo peitoral menor foi desinserido do processo coracoide para visualizar o nervo musculocutâneo e o ponto em que este penetra no músculo coracobraquial (Figura 2).

Todos os cadáveres eram do sexo masculino, com idades variando de 43 a 79 anos (média de 57 anos). Não foram levados em conta altura, peso, cor e raça de cada cadáver. Nenhum cadáver apresentava deficiência física ou cicatrizes nos ombros estudados.

Foram realizadas duas mensurações, tomando como referência o processo coracoide:

1) A distância da borda inferior do processo coracoide ao ponto de penetração do ramo mais proximal do nervo musculocutâneo no músculo coracobraquial (independente se este ramo era o principal ou o acessório).

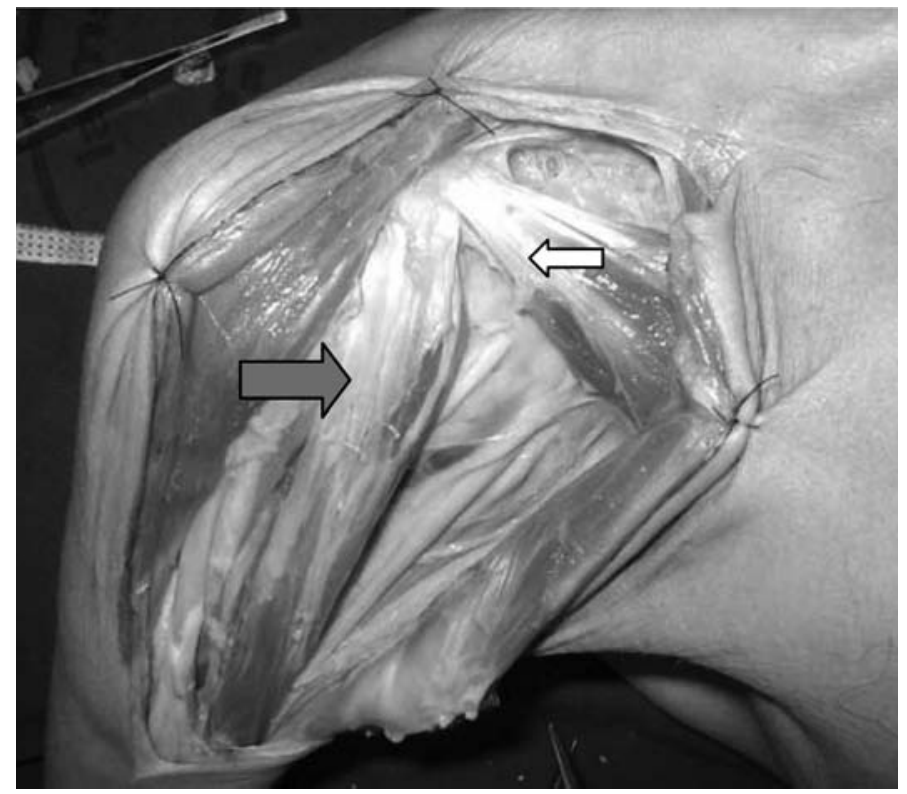

Figura 1 - Após desinserção do músculo peitoral maior $\diamond$ Músculo peitoral menor $\Rightarrow$ Músculo coracobraquial

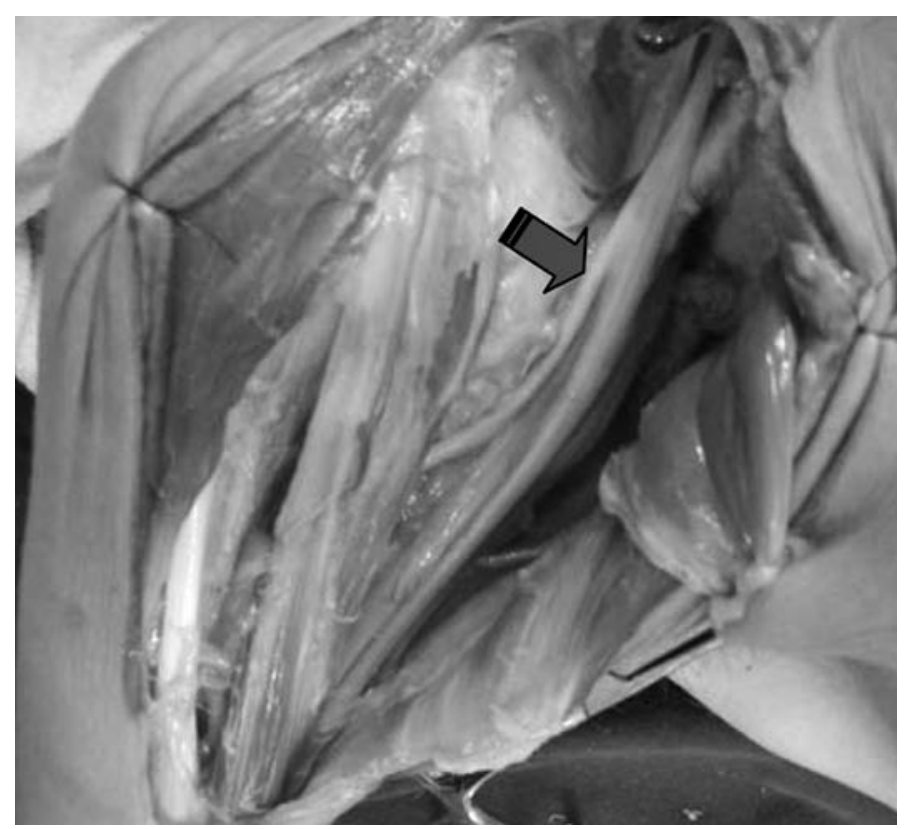

Figura 2 - Após desinserção do músculo peitoral podemos visualizar o nervo musculocutâneo (seta)

Esta distância foi denominada de base;

2) A distância da borda inferomedial do processo coracoide ao ponto em que o fascículo lateral cruza a borda inferior do músculo subclávio. Esta distância foi denominada de altura;

Com estas duas medidas, foram realizados cálculos matemáticos para determinar a área triangular criada com outra linha imaginária unindo os dois pontos (Figura 3).

As duas medidas foram mensuradas em centímetros e a área triangular calculada em centímetros quadrados. 


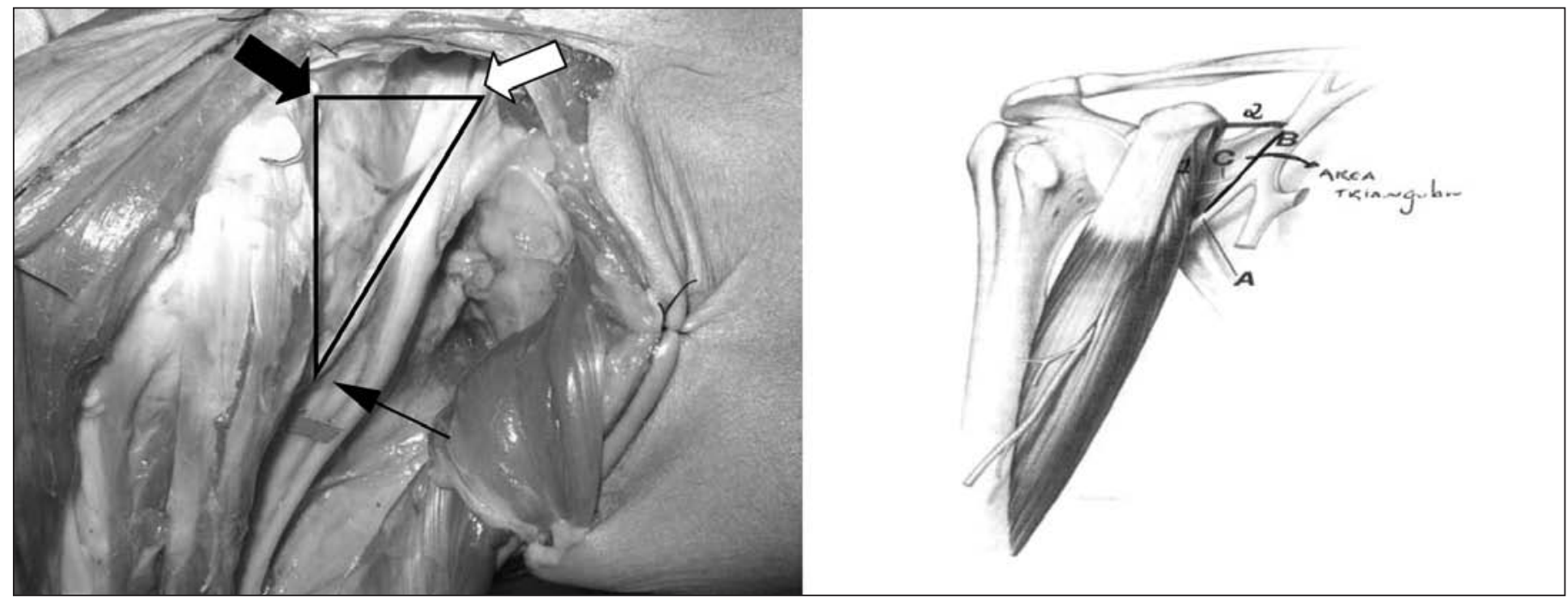

Figura 3 - Área triangular Processo coracoide (seta preta pequena). Fascículo lateral (seta branca). Nervo musculocutâneo (seta preta estreita).

\section{RESULTADOS}

Os resultados obtidos e a análise estatística estão descritos nas Tabelas 1 e 2.

Tabela 1 - Resumo das medidas realizadas com os valores de média, desvio padrão, mediana, mínimo, máximo e intervalo de confiança das medidas de base, altura e área dos 20 cadáveres estudados

\begin{tabular}{c|c|c|c|c}
\hline & & Base & Altura & Área \\
\hline & Média & 3,42 & 2,75 & 4,92 \\
\hline & Desvio padrão & 0,59 & 0,71 & 1,95 \\
\hline & Mediana & 3,47 & 2,85 & 5,30 \\
\hline & Mínimo & 2,38 & 1,03 & 1,22 \\
\hline IC a & Máximo & 4,30 & 3,80 & 7,99 \\
\hline $95 \% *$ & LI & 3,17 & 2,44 & 4,07 \\
\hline
\end{tabular}

*IC: intervalo de confiança para 95\% da população estudada; LI: limite inferior do intervalo de confiança; LS: limite superior do intervalo de confiança.

Tabela 2 - Comparação entre os lados mensurados para as medidas de base, altura e área

\begin{tabular}{|c|c|c|c|c|c|c|c|c|c|}
\hline \multirow{2}{*}{ Variável } & \multirow{2}{*}{ Lado } & \multirow{2}{*}{ Média } & \multirow{2}{*}{ DP } & \multirow{2}{*}{ Mediana } & \multirow{2}{*}{ Mínimo } & \multirow{2}{*}{ Máximo } & \multicolumn{2}{|c|}{ IC a $95 \%$ * } & \multirow{2}{*}{$\mathrm{p}^{* *}$} \\
\hline & & & & & & & LI & LS & \\
\hline \multirow{2}{*}{ Base } & Direito & 3,25 & 0,56 & 3,12 & 2,38 & 4,30 & 2,90 & 3,59 & 0,721 \\
\hline & Esquerdo & 3,60 & 0,58 & 3,73 & 2,68 & 4,24 & 3,24 & 3,96 & \\
\hline \multirow{2}{*}{ Altura } & Direito & 2,58 & 0,76 & 2,54 & 1,03 & 3,53 & 2,11 & 3,05 & 0,333 \\
\hline & Esquerdo & 2,93 & 0,65 & 3,00 & 1,77 & 3,80 & 2,53 & 3,33 & \\
\hline \multirow{2}{*}{ Área } & Direito & 4,35 & 1,89 & 3,97 & 1,22 & 7,52 & 3,18 & 5,53 & 0,508 \\
\hline & Esquerdo & 5,49 & 1,93 & 5,77 & 2,37 & 7,99 & 4,29 & 6,69 & \\
\hline
\end{tabular}

*IC: intervalo de confiança para $95 \%$ da população estudada; LI: limite inferior do intervalo de confiança; LS: limite superior do intervalo de confiança.

**: nível descritivo de probabilidade do teste não-paramétrico de Wilcoxon

\section{ANÁLISE ESTATÍSTICA}

Inicialmente todas as variáveis foram analisadas descritivamente. Para as variáveis quantitativas esta análise foi feita através da observação dos valores mínimos e máximos, e do cálculo de médias, desvios padrão, medianas e cálculo do intervalo de confiança a 95\%.

Para a análise da hipótese de igualdade das medidas realizadas nos lados direito e esquerdo utilizou-se o teste não-paramétrico de Wilcoxon.

O nível de significância utilizado para os testes foi de $5 \%$.

BASE - A distância da borda inferior do processo coracoide ao ponto de penetração do ramo mais proximal do nervo musculocutâneo no músculo coracobraquial variou de 2,38 a $4,30 \mathrm{~cm}$, com média de $3,42 \mathrm{~cm}$. O desvio padrão foi de $0,59 \mathrm{~cm}$. O estudo comparativo entre os lados direito e esquerdo demonstrou que a diferença entre as médias das medidas é $0,35 \mathrm{~cm}(\mathrm{p}=0,721)$.

ALTURA - A distância partindo da borda inferomedial do processo coracoide, ao ponto em que o fascículo lateral cruza a borda inferior do músculo subclávio variou de 1,03 a 3,80cm, com média de $2,75 \mathrm{~cm}$. O desvio padrão foi de $0,71 \mathrm{~cm}$. O estudo comparativo entre os lados direito e esquerdo demonstrou que a diferença entre as médias das medidas é de $0,35 \mathrm{~cm}(\mathrm{p}=0,333)$.

ÁREA - A área triangular encontrada variou de 1,22 a $7,99 \mathrm{~cm}^{2}$, com média de $4,92 \mathrm{~cm}^{2}$. O desvio padrão foi de $1,95 \mathrm{~cm}^{2}$. O estudo comparativo entre os lados direito e esquerdo demonstrou que a diferença entre as médias das medidas é $1,14 \mathrm{~cm}^{2}(\mathrm{p}=0,508)$. 


\section{DISCUSSÃO}

As relações anatômicas do nervo musculocutâneo vêm sendo estudadas ao longo dos últimos anos afim de evitar lesões durante procedimentos cirúrgicos do ombro. Helfet $^{(9)}$ foi o primeiro a reconhecer a existência de ramos menores do nervo musculocutâneo, que penetram o músculo coracobraquial proximal ao ramo principal. Este autor observou, em 1958, localização mais proximal do nervo musculocutâneo no músculo coracobraquial em relação ao processo coracoide.

Klepps et al $^{(10)}$ relataram dois casos de neuropraxia do nervo musculocutâneo durante procedimentos de transferência do músculo peitoral maior para o tubérculo menor, nos casos de rotura do tendão do músculo subescapular, em que um dos casos obteve resolução espontânea e o outro foi submetido à exploração, sendo evidenciado tensionamento do nervo musculocutâneo, que foi solucionado com alteração da técnica de reinserção do músculo peitoral maior com alívio da compressão do nervo.

Flatow et $a l^{(6)}$ e Klepps et $a l^{(10)}$ estudaram as relações anatômicas do nervo musculocutâneo e mediram a distância do processo coracoide ao ponto de penetração do nervo musculocutâneo no músculo coracobraquial, enfatizando a presença de pequenos ramos que penetram o músculo mais proximal do que o ramo principal, sendo em alguns casos os responsáveis pelas complicações encontradas.

O ramo principal do nervo musculocutâneo penetra o músculo coracobraquial em $92 \%$ dos casos com média de distância do processo coracoide ao ponto de penetração de 5,6cm; já os ramos menores apresentaram média de distância de $3,1 \mathrm{~cm}^{(5)}$.

Para Klepps et $a^{(10)}$, o ramo principal penetra a $6,1 \mathrm{~cm}$ do processo coracoide, enquanto que os ramos menores penetram a $4,4 \mathrm{~cm}$.

Em nosso estudo, não levamos em consideração o ramo principal para a realização das medidas. Mensuramos a distância da borda inferior do processo coracoide ao ponto de penetração do ramo mais proximal do nervo musculocutâneo no músculo coracobraquial (base), que variou de 2,38 a 4,30cm, com média de $3,42 \mathrm{~cm}$, sendo o desvio padrão de $0,59 \mathrm{~cm}$. Não encontramos, na literatura consultada, estudos que mensurem a área e a altura. A medida da altura foi em média de $2,75 \mathrm{~cm}$, variando entre 1,03 a 3,80cm e a média da área foi de $4,92 \mathrm{~cm}^{2}$, variando entre 1,22 a $7,99 \mathrm{~cm}^{2}$ (intervalo de confiança para $95 \%$ da população estudada). Não foi encontrada diferença significativa entre os lados direito e esquerdo.

\section{CONCLUSÃO}

Concluímos que o nervo musculocutâneo, durante as abordagens cirúrgicas do ombro, está situado entre 2,38 a 4,30cm distal e 1,03 a 3,80 cm medial à borda inferior do processo coracoide. Estas mensurações são importantes e devem ser respeitadas durante as abordagens cirúrgicas desta topografia para evitar lesão do nervo musculocutâneo. Acreditamos que este estudo possa ajudar na prevenção de lesões do nervo musculocutâneo durante procedimentos cirúrgicos.

\section{REFERÊNCIAS}

1. Latarjet A, Testut L. Tratado de anatomia humana. São Paulo: Salvat; 1976. p. 269-72.

2. Bach BR Jr, O'Brien SJ, Warren RF, Leighton M. An unusual neurological complication of the Bristow procedure. A case report. J Bone Joint Surg Am. 1988;70(3):458-60.

3. Brav EA. An evaluation of the Putti-Platt reconstruction procedure for recurrent dislocation of the shoulder. J Bone Joint Surg Am. 1955;37(4):731-41.

4. Crenshaw AH Jr. Surgical techniches and approaches. In: Canale ST. Campbell's operative orthopaedics. 10th edition. St. Louis: Mosby; 2003. p. 3-126.

5. DePalma AF. Regional, variational, surgical anatomy. In: DePalma AF. Surgery of the shoulder. 3rd ed. Philadelphia: Lippincott; 1983. p. 35-64.

6. Flatow EL, Bigliani LU, April EW. An anatomic study of the musculocuta-

neous nerve and its relationship to the coracoid process. Clin Orthop Relat Res. 1989;(244):166-71.

7. Galatz LM, Connor PM, Calfee RP, Hsu JC, Yamaguchi K. Pectoralis major transfer for anterior-superior subluxation in massive rotator cuff insufficiency. J Shoulder Elbow Surg. 2003;12(1):1-5.

8. Hardy M. Basic anatomy of the shoulder and elbow. In: Inglis AE. Symposium on total joint replacement of the upper extremity. St. Louis: Mosby; 1982.

9. Helfet AJ. Coracoid transplantation for recurring dislocation of the shoulder. J Bone Joint Surg Br. 1958;40(2):198-202.

10. Klepps SJ, Goldfarb C, Flatow E, Galatz LM, Yamaguchi K. Anatomic evaluation of the subcoracoid pectoralis major transfer in human cadavers. J Shoulder Elbow Surg. 2001;10(5):453-9. 